\title{
Engineering of a genome-reduced host: practical application of synthetic biology in the overproduction of desired secondary metabolites
}

\author{
Hong Gao ${ }^{1 *}$, Ying Zhuo ${ }^{1,2 *}$, Elizabeth Ashforth ${ }^{1}$, Lixin Zhang ${ }^{1 凶}$ \\ ${ }^{1}$ CAS Key Laboratory of Pathogenic Microbiology \& Immunology, Institute of Microbiology, Chinese Academy of Sciences \\ (CAS), Beijing 100101, China. \\ 2 Graduate University of Chinese Academy of Sciences, Beijing 100049, China. \\ $\triangle$ Correspondence: Izhang03@gmail.com \\ Received May 19, 2010 Accepted May 28, 2010
}

\begin{abstract}
Synthetic biology aims to design and build new biological systems with desirable properties, providing the foundation for the biosynthesis of secondary metabolites. The most prominent representation of synthetic biology has been used in microbial engineering by recombinant DNA technology. However, there are advantages of using a deleted host, and therefore an increasing number of biotechnology studies follow similar strategies to dissect cellular networks and construct genomereduced microbes. This review will give an overview of the strategies used for constructing and engineering reduced-genome factories by synthetic biology to improve production of secondary metabolites.
\end{abstract}

KEYWORDS synthetic biology, reduced-genome, secondary metabolite

\section{INTRODUCTION}

Secondary metabolites are an extremely diverse and important class of natural products, including clinically important antibiotic, anticancer, and antiviral compounds along with agents utilized in agricultural, veterinary, and food industries (Gao et al., 2010). Approximately 23,000 bioactive secondary metabolites produced by microorganisms have been reported and approximately 150 of them are routinely used in pharmacology, agriculture or other fields (Bérdy, 2005). Recent advances in the synthetic biology of these secondary metabolite producing organisms have allowed for higher levels of production and biosynthesis of novel products.

Synthetic biology can be defined as the "re-purposing and re-design of biological systems for novel purposes or applications" (Marner, 2009). Synthetic biology can be applied to transfer the ability of secondary metabolite biosynthesis from source organisms to a genetically friendlier host. What separates the current incarnation of synthetic biology from the genetic engineering or metabolic engineering of the past is an emphasis on principles from engineering such as modularity, standardization, and rigorously predictive models. As such, synthetic biology represents a new paradigm for learning about and using secondary metabolites and data, with applications in biotechnology (Agapakis and Silver, 2009).

The genome-reduced heterologous host, named "chassis," should be more effective for biosynthesis of the desired products as it supplies only the necessary genes for full functionality, and therefore reduces the complexity of the metabolic network. Recently, with the vast increase in annotated genome information and high-throughput technologies, increasing number of genome-reduces hosts have been developed and studied (Koonin, 2003; Gil et al., 2004; Fujio, 2007).

In this review we focus on the advances in the study of genome-reduced hosts and their potential in synthetic biology

"These authors contributed equally to the work. 
frameworks to improve the biosynthesis of secondary metabolites.

\section{IMPROVEMENT OF SECONDARY METABOLITE PRODUCTION}

A great effort is underway to improve the production of secondary metabolites, including manipulation of native producers (Zhuo et al., 2010) and development of heterologous hosts (Chen et al., 2010). Similar molecular biologic techniques are used for native and heterologous hosts, including improving flux through the biosynthetic pathway, enhancing precursor supply, and reducing by-product formation (Rokem et al., 2007).

There are many successful examples of manipulation in native producers to improve the production of secondary metabolites (Rokem et al., 2007). However, intrinsic shortcomings in this method exist.

i) Many bioactive secondary metabolite producers are difficult to cultivate, slow growing or even uncultivable (Reichenbach, 2001; Harada, 2004).

ii) Some microorganisms, producing important secondary metabolites, are not possible to efficiently genetically engineer. For example, spinosad is a bioinsecticide produced by Saccharopolyspora spinosa (Jin et al., 2009). This strain has been reported to be highly recalcitrant to transformation efforts because it expresses an unusually effective set of restriction systems to protect it from bacteriophages and other foreign DNA (Matsushima and Baltz, 1994; Matsushima et al., 1994). Although protoplast and conjugation transformation systems for S. spinosa were developed in the early 1990 s and were applied in the genetic engineering of $S$. Spinosa (Huang et al., 2009), the manipulation success rate was not particularly high.

iii) Because of the complexities of pathway networks in microorganisms, it is difficult to obtain desired results by manipulating multiple regulators. For example, C-1027 belongs to the enediyne family, a class of compounds that are well known for their extreme cytotoxicities, and whose members have the potential to be developed into anticancer drugs (Horsman et al., 2009). Three putative regulatory genes, $s g c R 1, s g c R 2$ and $s g c R 3$, were found in the C-1027 biosynthesis gene cluster (Liu et al., 2002). When sgcR1, $s g c R 2$ and $s g c R 3$ were overexpressed separately, a noticeable increase in $\mathrm{C}-1027$ production was observed (Wang et al., 2009; Chen et al., 2010). However, placing the three activator genes in tandem on a high-copy-number plasmid under the control of ErmE $E^{\star}$ promoter and overexpressing them together, failed to further increase the production of C1027 (Chen et al., 2010). The consequence of the first two problems is an increased interest in heterologous production of secondary metabolite pathways in new fast-growing hosts where genetic tools are available. The third problem, however, still remains unsolved.
In nature, natural biologic systems appear to continuously evolve to meet the requirement of existence and replication. Not surprisingly, the absolute performance of naturally evolved systems sometimes exceeds that produced by human-directed designs (Aho et al., 1988). Despite decades of scientific progress, we still have only a partial, and in some cases ambiguous understanding of how the parts that comprise the cell work together to encode a functional whole, a vital constituent of purpose-orientation engineering research.

Deletion of large blocks of non-essential genes has been found to reduce the production of unwanted by-products, increase genome stability, and streamline metabolic pathyway of interest by reducing unnecessary metabolic pathways and complex regulatory networks (Mizoguchi et al., 2008; Lee et al., 2009). With the recent increase in sequencing and the completion of several microbial genomes, functional genes can be identified with the modeling of comparative and functional analysis data (Fig. 1). To understand and manipulate these evolved and complicated systems, genomereduction by planned, precise deletion has been carried out, thus tackling the third problem of increasing secondary metabolite yield (Mizoguchi et al., 2007).

\section{STRATEGIES FOR ENGINEERING REDUCED-GENOME HOSTS}

Microbes have numerous genes that are not essential under normal cultivation conditions, and are rather wasteful in terms of energy consumption. The non-essential genes and sequences, including recombinogenic or mobile DNA and cryptic virulence genes, were eliminated in Escherichia coli (Pósfai et al., 2006). The multiple-deletions, up to $15 \%$ of the total genome, did not show any detrimental effect and preserved good growth profiles and protein production in the strain.

Genome reductions may decrease the redundancy among genes and regulatory circuits and could also lead to unanticipated beneficial properties, such as, genome stabilization (Pósfai et al., 2006), high electroporation efficiency, and accurate propagation of recombinant genes and plasmids that are unstable in other strains. It also provided the possibility to improve expression of exogenous metabolic pathways (Chakiath and Esposito, 2007). Both increased stability in the host and improved efficiency of recombination are ideal outcomes for the production of secondary metabolites.

Currently, there are two main approaches to obtain a genome-reduced cell: chemical synthesis of the specialized genome; and genome size reduction by deletion. The first example of an artificially synthesized genome was the 7440 bp poliovirus DNA (Cello et al., 2002). While the time and monetary cost of, DNA synthesis has been reduced significantly (Tian et al., 2004), the genome of bacteria is still 


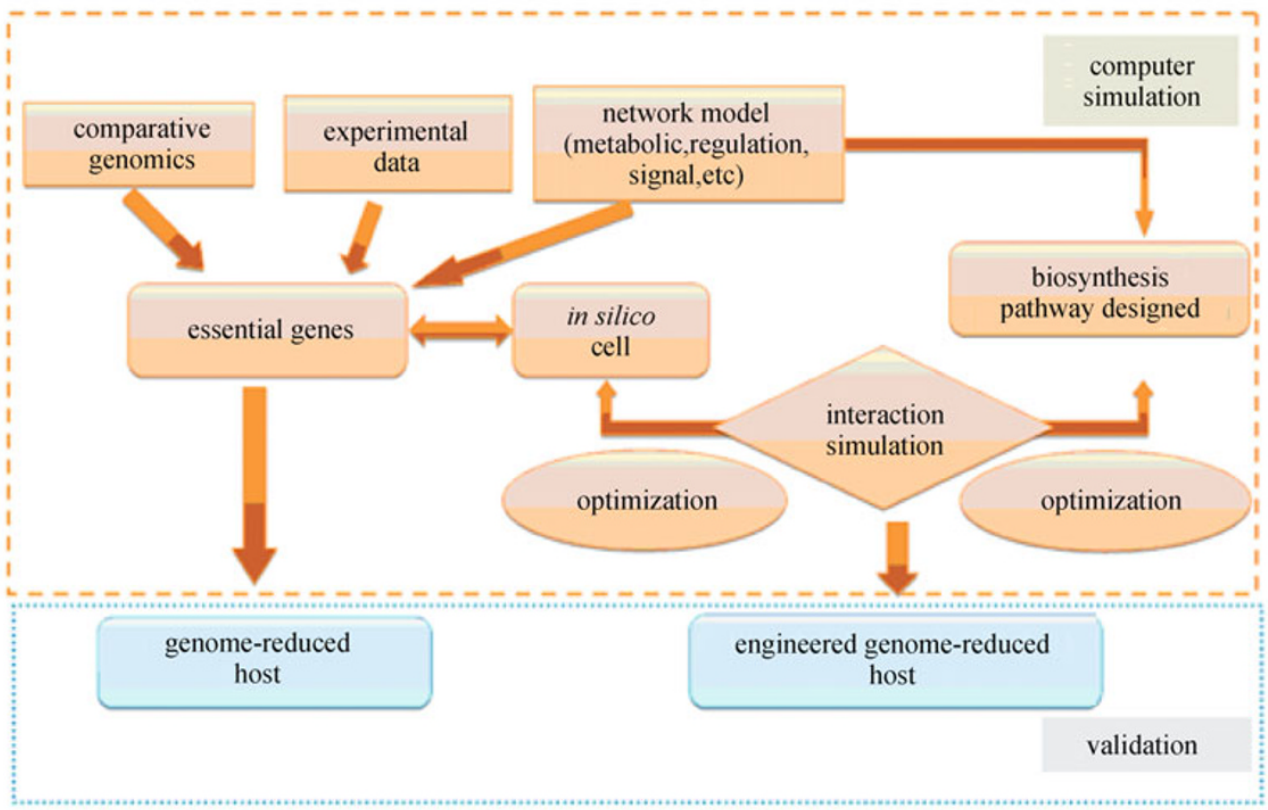

Figure 1. The schematic diagram of the process to construct and engineer genome-reduce host. The essential genes are determined by combination of comparative genomics, experimental data and models including metabolic, regulation and signal network models. Then, genome-reduced host is constructed by deleting the nonessential genes. For introduction of biosynthesis pathway designed, interaction between the biosynthesis pathway and the 'background genes' in the genome-reduced host is simulated in silico, and after several cycles of optimization and simulation, an engineered genome-reduced host with desired functionality is obtained. Finally, the host is subjected to experiments to be validated.

too large to be synthesized (Fraser et al., 1995). And with current technology, it would be difficult to activate the synthesized genome as a replicable chromosome, in a living cell (Mizoguchi et al., 2007). On the other hand, there are many examples of applying a reduction to the characteristics related to metabolites biosynthesis (Table 1). These reports demonstrated the potential of genome engineering for creating a simpler and more efficient cell. Compared with genome synthesis, reduction approaches would seem to be a quicker and more realistic way to construct a genomereduced host with the potential application of biosynthesis of natural products.
Many of the examples of genome-reduced hosts, as shown in Table 1, were constructed to increase the production of primary metabolites, such as amino acid and ethanol. There have also been reports that the genome-reduced hosts can be engineered to further improve the production of natural products. Lee et al. overexpressed a feedback-resistant threonine operon $\left(t h r A^{*} B C\right)$, deleted threonine dehydrogenase and threonine transporters encoding genes $(t d h, t d c C$ and sstT), and introduced a mutant threonine exporter (rhtA23) in a genome-reduced E. coli strain. Compared with the wild-type strain, production of L-threonine, essential for growth and maintenance of commercial livestock (Debabov,

Table 1 Characteristics of genome-reduced hosts

\begin{tabular}{|c|c|c|c|}
\hline strain & deletion size & characteristic & references \\
\hline \multicolumn{4}{|l|}{ Escherichia coli } \\
\hline MDS42 & $663.3 \mathrm{~kb}(14.30 \%)$ & higher electroporation efficiency & Pósfai et al., 2006 \\
\hline MGF-01 & $1.03 \mathrm{Mb}(22 \%)$ & increased threonine production (2-fold) & Mizoguchi et al., 2007 \\
\hline \multicolumn{4}{|l|}{ Bacillus subtilis } \\
\hline MGB874 & $873.5 \mathrm{~kb}(20.7 \%)$ & $\begin{array}{l}\text { increased productivity of extracellular } \\
\text { cellulose ( } 1.7 \text {-fold }) \text { and protease ( } 2.5 \text {-fold) }\end{array}$ & Morimoto et al., 2008 \\
\hline S. cerevisiae & $531.5 \mathrm{~kb}(5 \%)$ & $\begin{array}{l}\text { increased production of ethanol (1.8-fold) } \\
\text { and glycerol (2-fold) }\end{array}$ & Murakami et al., 2007 \\
\hline Streptomyces avermitilis & $1.67 \mathrm{Mb}(18.54 \%)$ & $\begin{array}{l}\text { increased production of streptomycin (4-fold) } \\
\text { and cephamycin C (2-fold) }\end{array}$ & Komatsu et al., 2010 \\
\hline
\end{tabular}


2003 ), in the resulting mutant was increased by $83 \%$ (Lee et al., 2009).

\section{SYNTHESIS BIOLOGY OF SECONDARY METABOLITES IN REDUCED-GENOME HOST}

In Table 1, Streptomyces avermitilis is the only example of a genome-reduced host used to biosynthesize secondary metabolites. With non-pathogenicity and a well-established fermentation technology, Streptomyces are considered attractive candidates for the production of heterologous proteins for several reasons, non-withstanding their efficient secretion mechanism by which proteins are localized in the culture supernatant (Brawner et al., 1991; Anné and Van Mellaert, 1993).

Comparative analysis of three published Streptomyces genomes, S. avermitilis, S. coelicolor A3(2) and $S$. griseus, revealed a conserved core region of $6.28-6.50 \mathrm{Mb}$ in which the genes essential for growth are located (Ohnishi et al., 2008). The left and right subtelomeric regions contain strainspecific genes as well as genes encoding secondary metabolite biosynthesis but no essential genes. Therefore, removal of the large subtelomeric regions of $S$. avermitilis would not affect either their growth or primary metabolism. The industrial microorganism, S. avermitilis, was constructed as a versatile model host for heterologous expression of genes encoding secondary metabolite biosynthesis, by systematically removing non-essential genes in this way (Komatsu et al., 2010). The multiple-deletion series strain, with up to $18.6 \%$ of the wild-type chromosome removed, did not produce any of the major endogenous secondary metabolites and was successfully used as the hosts for efficient expression of foreign metabolites. Three different exogenous biosynthetic gene clusters encoding the biosynthesis of streptomycin, cephamycin C and pladienolide, were efficiently expressed in individual transformants, at levels higher than those of the native-producing species.

The plant terpenoid intermediate, amorpha-4,11-diene, can also be exogenously expressed in mutants, optimized for terpenoid production, by introduction of a synthetic gene optimized for Streptomyces codon usage (Komatsu et al., 2010). These examples highlight the strength and flexibility of engineered $S$. avermitilis as a model host for heterologous expression of secondary metabolites, and illustrate its suitability for biosynthesis of non-natural metabolites.

\section{CONCLUSION AND FUTURE PERSPECTIVES}

Growing demand to increase the production of economically important secondary metabolites via programmable and robust biological systems, is the main driving force behind the synthetic biology of genome-reduced hosts. To engineer these desired hosts, we must characterize the non-essential genes (which vary with the desired product and cultivation conditions), obtain genome-reduced strains containing only the essential genes, analyze the biosynthesis pathway of specific secondary metabolites and the necessary cofactors thereof, assemble the biosynthesis pathway of desired secondary metabolites by picking optimal protein modules, and transfer the pathway into a suitable genome-reduced host efficiently. The concepts of genome-reduce hosts and the procedures for engineering the hosts to improve the production of secondary metabolites discussed here provide incentives for further developments in synthetic biology.

Other than the biosynthesis of secondary metabolites, synthetic biology could be applied to many fields, including biofuel, biomedicine, etc. However, three key challenges for a wide implementation of genome-reduced hosts in synthetic biology currently exist:

i) Undefined non-essential genes: non-essential genes vary with desired function and culture conditions, therefore general knowledge of non-essential genes and rules to distinguish non-essential genes from essentials ones are needed to avoid repeated work defining non-essential genes in specific environments.

ii) Compatibility: in this system discussed, synthetic biology is used to introduce the biosynthesis pathway of secondary metabolites into the genome-reduced host, and if necessary further engineered to improve the production of desired products. However, in these processes, unexpected interactions can occur between introduced genes and "background genes" within the host genome (Kwok, 2010). A comprehensive understanding of the metabolic pathway of the host would help to resolve this problem.

iii) The choice of suitable host for specific products: currently, most researches on genome-reduced have focused on $E$. coli, because $E$. coli is the most studied and wellunderstood microorganisms (Sharma et al., 2007). However, characterization of the complex, branching, and often interactive metabolic pathways of many organisms is feasible with advances in-omics analysis and may provide more suitable starting platforms for improving of secondary metabolite production, such as a Streptomyces host.

In the framework of synthetic biology, genome-reduced hosts will become increasingly important to improve the production of secondary metabolites. In-depth exploration of the global metabolic pathways of hosts is likely to provide a solid theoretical foundation of the biosynthesis of secondary metabolites, thus obtaining a highly efficient and physiologically robust strain, and therefore ensuring their successful applications in biotechnology industries.

\section{ACKNOWLEDGEMENTS}

This work was supported in part by grants from the National Programs for High Technology Research and Development Program (863 Program) (Grant Nos. 2006AA09Z402, 2007AA09Z443), the National Basic Research Program of China (973 Program) (Grant No. 2004CB719601), National Natural Science Foundation of China 
(Grant Nos. 30560001, 30600001, 30700015), National Key Technology R\&D Program (2007BAI26B02), the National Science \& Technology Pillar Program (No. 200703295000-02), Important National Science \& Technology Specific Projects (Nos. 2008ZX09401-005, 2009ZX09302-004), Science and Technology Planning Project of Guangdong Province, China (No. 2006A50103001), Key Project of International Cooperation (2007DFB31620). L.-X. Z. received funding from the Hundred Talents Program.

\section{REFERENCES}

Agapakis, C.M., and Silver, P.A. (2009). Synthetic biology: exploring and exploiting genetic modularity through the design of novel biological networks. Mol Biosyst 5, 704-713.

Aho, A.C., Donner, K., Hydén, C., Larsen, L.O., and Reuter, T. (1988). Low retinal noise in animals with low body temperature allows high visual sensitivity. Nature 334, 348-350.

Anné, J., and Van Mellaert, L. (1993). Streptomyces lividans as host for heterologous protein production. FEMS Microbiol Lett 114, 121-128.

Bérdy, J. (2005). Bioactive microbial metabolites. J Antibiot (Tokyo) $58,1-26$.

Brawner, M., Poste, G., Rosenberg, M., and Westpheling, J. (1991). Streptomyces: a host for heterologous gene expression. Curr Opin Biotechnol 2, 674-681.

Cello, J., Paul, A.V., and Wimmer, E. (2002). Chemical synthesis of poliovirus cDNA: generation of infectious virus in the absence of natural template. Science 297, 1016-1018.

Chakiath, C.S., and Esposito, D. (2007). Improved recombinational stability of lentiviral expression vectors using reduced-genome Escherichia coli. Biotechniques 43, 466, 468, 470.

Chen, Y., Smanski, M.J., and Shen, B. (2010). Improvement of secondary metabolite production in Streptomyces by manipulating pathway regulation. Appl Microbiol Biotechnol 86, 19-25.

Debabov, V.G. (2003). The threonine story. Adv Biochem Eng Biotechnol 79, 113-136.

Fraser, C.M., Gocayne, J.D., White, O., Adams, M.D., Clayton, R.A., Fleischmann, R.D., Bult, C.J., Kerlavage, A.R., Sutton, G., Kelley, J.M., et al. (1995). The minimal gene complement of Mycoplasma genitalium. Science 270, 397-403.

Fujio, T. (2007). Minimum genome factory: innovation in bioprocesses through genome science. Biotechnol Appl Biochem 46, 145-146.

Gao, H., Zhou, X., Gou, Z., Zhuo, Y., Fu, C., Liu, M., Song, F., Ashforth, E., and Zhang, L. (2010). Rational design for overproduction of desirable microbial metabolites by precision engineering. Antonie van Leeuwenhoek. In press.

Gil, R., Silva, F.J., Peretó, J., and Moya, A. (2004). Determination of the core of a minimal bacterial gene set. Microbiol Mol Biol Rev 68, 518-537.

Harada, K. (2004). Production of secondary metabolites by freshwater cyanobacteria. Chem Pharm Bull (Tokyo) 52, 889-899.

Horsman, G.P., Van Lanen, S.G., and Shen, B. (2009). Iterative type I polyketide synthases for enediyne core biosynthesis. Methods Enzymol 459, 97-112.

Huang, K.X., Xia, L., Zhang, Y., Ding, X., and Zahn, J.A. (2009). Recent advances in the biochemistry of spinosyns. Appl Microbiol Biotechnol 82, 13-23.
Jin, Z.H., Xu, B., Lin, S.Z., Jin, Q.C., and Cen, P.L. (2009). Enhanced production of spinosad in Saccharopolyspora spinosa by genome shuffling. Appl Biochem Biotechnol 159, 655-663.

Komatsu, M., Uchiyama, T., Omura, S., Cane, D.E., and Ikeda, H. (2010). Genome-minimized Streptomyces host for the heterologous expression of secondary metabolism. Proc Natl Acad Sci U S A 107, 2646-2651.

Koonin, E.V. (2003). Comparative genomics, minimal gene-sets and the last universal common ancestor. Nat Rev Microbiol 1, 127-136.

Kwok, R. (2010). Five hard truths for synthetic biology. Nature 463 , 288-290.

Lee, J.H., Sung, B.H., Kim, M.S., Blattner, F.R., Yoon, B.H., Kim, J.H., and Kim, S.C. (2009). Metabolic engineering of a reduced-genome strain of Escherichia coli for L-threonine production. Microb Cell Fact 8, 2.

Liu, W., Christenson, S.D., Standage, S., and Shen, B. (2002). Biosynthesis of the enediyne antitumor antibiotic C-1027. Science 297, 1170-1173.

Marner, W.D. 2nd. (2009). Practical application of synthetic biology principles. Biotechnol J 4, 1406-1419.

Matsushima, P., and Baltz, R. (1994). Transformation of Saccharopolysopora spinosa protoplasts with plasmid DNA modified in vitro to avoid host restriction. Microbiology 140, 139-143.

Matsushima, P., Broughton, M.C., Turner, J.R., and Baltz, R.H. (1994). Conjugal transfer of cosmid DNA from Escherichia coli to Saccharopolyspora spinosa: effects of chromosomal insertions on macrolide A83543 production. Gene 146, 39-45.

Mizoguchi, H., Mori, H., and Fujio, T. (2007). Escherichia coli minimum genome factory. Biotechnol Appl Biochem 46, 157-167.

Mizoguchi, H., Sawano, Y., Kato, J., and Mori, H. (2008). Superpositioning of deletions promotes growth of Escherichia coli with a reduced genome. DNA Res 15, 277-284.

Morimoto, T., Kadoya, R., Endo, K., Tohata, M., Sawada, K., Liu, S., Ozawa, T., Kodama, T., Kakeshita, H., Kageyama, Y., et al. (2008). Enhanced recombinant protein productivity by genome reduction in Bacillus subtilis. DNA Res 15, 73-81.

Murakami, K., Tao, E., Ito, Y., Sugiyama, M., Kaneko, Y., Harashima, S., Sumiya, T., Nakamura, A., and Nishizawa, M. (2007). Large scale deletions in the Saccharomyces cerevisiae genome create strains with altered regulation of carbon metabolism. Appl Microbiol Biotechnol 75, 589-597.

Ohnishi, Y., Ishikawa, J., Hara, H., Suzuki, H., Ikenoya, M., Ikeda, H., Yamashita, A., Hattori, M., and Horinouchi, S. (2008). Genome sequence of the streptomycin-producing microorganism Streptomyces griseus IFO 13350. J Bacteriol 190, 4050-4060.

Pósfai, G., Plunkett, G. 3rd, Fehér, T., Frisch, D., Keil, G.M., Umenhoffer, K., Kolisnychenko, V., Stahl, B., Sharma, S.S., de Arruda, M., et al. (2006). Emergent properties of reduced-genome Escherichia coli. Science 312, 1044-1046.

Reichenbach, H. (2001). Myxobacteria, producers of novel bioactive substances. J Ind Microbiol Biotechnol 27, 149-156.

Rokem, J.S., Lantz, A.E., and Nielsen, J. (2007). Systems biology of antibiotic production by microorganisms. Nat Prod Rep 24,1262-1287.

Sharma, S.S., Blattner, F.R., and Harcum, S.W. (2007). Recombinant protein production in an Escherichia coli reduced genome strain. Metab Eng 9, 133-141.

Tian, J., Gong, H., Sheng, N., Zhou, X., Gulari, E., Gao, X., and 
Church, G. (2004). Accurate multiplex gene synthesis from programmable DNA microchips. Nature 432, 1050-1054.

Wang, L., Hu, Y., Zhang, Y., Wang, S., Cui, Z., Bao, Y., Jiang, W., and Hong, B. (2009). Role of sgcR3 in positive regulation of enediyne antibiotic C-1027 production of Streptomyces globisporus C-1027.
BMC Microbiol 9, 14.

Zhuo, Y., Zhang, W., Chen, D., Gao, H., Tao, J., Liu, M., Gou, Z., Zhou, X., BC, Y., Zhang, Q., et al. (2010). Reverse biological engineering of hrdB to enhance the production of avermectins in an industrial strain of Streptomyces avermitilis. Proc Natl Acad Sci U S A. In press. 